\title{
Correlation Co-Efficient Studies of Oil, Yield and Related Traits in Bt and Non Bt Hybrids
}

\author{
Nagappa Harijan* and B.M. Khadi
}

Seed Unit University of Agricultural Sciences, Dharwad, Karnataka, India

*Corresponding author

A B S T R A C T

\section{Keywords \\ Correlation, Cotton, Association and cotton oil \\ Article Info \\ Accepted: \\ 04 February 2018 \\ Available Online: \\ 10 March 2018}

Cotton has a proud place among the crops from earliest times. Apart from seed cotton the cotton seed oil also has very good quality and vitamin E. The oil content statistically positively correlation with seed cotton yield, seed index, bolls per plant, boll weight and sympodial branches. Monopodial branches exhibit no significant association with oil content.

\section{Introduction}

Cotton has a pride place among the crops from the earliest times. It finds mention in the Rigveda, the oldest scripture of Hindus. Manu, the law giver also referred to it in his Dharmashashtra. It was the excellence of Indian cotton fabrics famed as 'Webs of Woven Wind" which compelled European countries to seek new trade routes with India. Despite the advent of a multitude of other fibres, cotton, the white gold rules the world of textile. Cotton though mainly grown for fibre is also ranked as a major oilseed crop in the international market. Out of four major products i.e., meal, hull, oil and linters, oil is most important. Besides commercial importance it is used in the leather industry as a lubricant. Cotton seed oil can also be used for edible purpose after refining. Cotton seed oil is premium quality oil as it has no cholesterol and is a vegetable oil.

India is addressing the need for increased $\mathrm{Bt}$ cotton cultivars. These insect protected cotton varieties contain a naturally occurring substance, Bacillus thuringiensis (Bt) protein which has been used as an ingredient in safe and effective biological sprays for more than 50 years. Bt trait has been successfully transferred into several Indian lines. Extensive and fully replicated field traits of Bt cotton were conducted from 1998 to 2001 cropping seasons, meeting the government requirements for commercialization. Three $\mathrm{Bt}$ cotton cultivars have been approved for planting in 
India in 2002-03. Since, the introduction of Bt cotton hybrid around 44,500 ha were planted with three hybrids of Bt cotton in central and southern zones in 2002-03 season. This increased to some 1, 00,000 ha in 2003-04. In 2004-05 around four Bt cotton hybrids were planted over $5,00,000$ ha by three lakh resource poor farmers. With approval of 16 new hybrids of half a dozen companies including six Bt cotton hybrids for northern region, Bt cotton planting for 2005-06 season has experienced the highest yearly percentage growth rate increasing its area by 160 per cent (13 lakh ha). Around 10 lakh farmers elected to plant Bt cotton hybrids in northern, central and southern cotton growing zones of Indiaas compared to 3 lakh farmers in the previous year (Anon., 2006).

The knowledge of inter-character correlation of quantitative characters is useful to design an effective breeding programme. These correlations provide a reliable measure to differentiate the vital association useful in breeding from that of non-vital ones (Falconer, 1981).

The present investigation primarily aimed at assessment of inter-character association between oil and other seed properties viz., fibre properties. Character association was done in all the three experiments comprising of genetic stocks involving both tetraploid $\mathrm{Bt}$ and non-Bt commercial hybrids. The complete set of correlations among seed and oil related parameters are available in the genetic stock.

\section{Materials and Methods}

There are 36 hybrids $\mathrm{Bt}$ and non $\mathrm{Bt}$ counterpart hybrids viz.,JKCH-224,SDCH302, K-5308, KDCH-441, JK Indra, RCH2,JK Ishwar, JKCH-1947, RCH-134, JKCH22, Ankur-651 BGI, Ankur-651 BGI, RCH144, JKCH-1050, SBCH-311, PCH2270,NECH-3R, KDCHH-9810, NECN-2R,
RCH-118, JKCH-226, Dhruva, K-5316, KDCHH-9632, JK Varun, RCH-2171, RCH20, JK Durga, JKCH-99, RCH-138, JKCH1945, JK Gowri, RCH-377, VCH-111, NHH44, VICH-5, VICH-5 and VICH-9 have collected for correlation study of oil, yield and related traits. Phenotyping for oil content was done by Near Infrared Spectroscopy (NIRS) at all the three locations.

Soxhlet method for estimation of oil content: oil content was estimated by Soxhlet method as given by Jambunathan et al., (1985) with some modifications. $5 \mathrm{gms}$ of cotton seeds from each entry were powdered in a pestle and mortar. Cotton seed meal was extracted with petroleum ether for $5 \mathrm{hrs}$ approximately in a Soxhlet apparatus.

Petroleum ether was evaporated and the oil content was estimated by the difference in the weight between the two and was expressed in percentage. The phenotypic data obtained for oil content by this method was used for calibrating for oil in NIRS.

\section{Correlation coefficient}

Phenotypic and genotypic correlation coefficients between different variables were calculated by using covariance technique (Falconer, 1981). The analysis of covariance by following the method described by Singh and Choudhary (1977) is given below.

$$
\operatorname{Cov}_{\mathrm{xy}(\mathrm{g})}=\frac{\mathrm{TMSP}-\mathrm{EMSP}}{\mathrm{r}}
$$

$\operatorname{Cov}_{\mathrm{xy}(\mathrm{e})}=\mathrm{EMSP}$

$\operatorname{Cov}_{x y}(p)=\operatorname{cov}_{x y}(g)+\operatorname{cov}_{x y}$

Genotypic correlation $=\mathrm{r}_{\mathrm{xy}(\mathrm{g})}=\operatorname{cov}_{\mathrm{xy}(\mathrm{g})} /\left[\mathrm{v}_{\mathrm{x}(\mathrm{g})}\right.$ $\left.\mathrm{x}_{\mathrm{y}(\mathrm{g})}\right]^{1 / 2}$

Phenotypic correlation $=\mathrm{r}_{\mathrm{xy}(\mathrm{p})}=\operatorname{cov}_{\mathrm{xy}(\mathrm{P})} /$ $\left[\mathrm{v}_{\mathrm{x}(\mathrm{p})} \times \mathrm{v}_{\mathrm{y}(\mathrm{p})}\right]^{1 / 2}$ 
Environmental correlation $=\mathrm{r}_{\mathrm{xy}(\mathrm{e})}=\operatorname{cov}_{\mathrm{xy}}(\mathrm{e}) /$ $\left[\mathrm{v}_{\mathrm{x}(\mathrm{e})} \mathrm{X} \mathrm{v}_{\mathrm{y}(\mathrm{e})}\right]^{1 / 2}$

Where, RMSP = replication mean sum of products, TMSP $=$ treatment mean sum of products, $\mathrm{EMSP}=$ error mean sum of products, $\operatorname{Cov}_{\mathrm{xy}}(\mathrm{g})=$ genotypic covariance between characters $\mathrm{x}$ and $\mathrm{y}, \operatorname{Cov}_{\mathrm{xy}}(\mathrm{p})=$ phenotypic covariance between characters $\mathrm{x}$ and $\mathrm{y}, \operatorname{Cov}_{\mathrm{xy}}(\mathrm{e})=$ error covariance between characters $\mathrm{x}$ and $\mathrm{y}, \mathrm{v}_{\mathrm{x}(\mathrm{g})}=$ genotypic variance of character $\mathrm{x}, \mathrm{v}_{\mathrm{y}(\mathrm{g})}=$ genotypic variance of character $\mathrm{y}, \mathrm{v}_{\mathrm{x}(\mathrm{p})}=$ phenotypic variance of character $\mathrm{x}, \mathrm{v}_{\mathrm{y}(\mathrm{p})}=$ phenotypic variance of character $\mathrm{y}, \mathrm{v}_{\mathrm{x}(\mathrm{e})}=$ error variance of character $\mathrm{x}, \mathrm{v}_{\mathrm{y}(\mathrm{e})}=$ error variance of character $\mathrm{y}$

Genotypic $\left(\mathrm{r}_{\mathrm{g}}\right)$, Phenotypic $\left(\mathrm{r}_{\mathrm{p}}\right)$ and Environmental $\left(r_{e}\right)$ correlation coefficients among different characters were estimated for which variance ratio was significant from the variance and covariance components following the method given by Hayes et al., (1955).

Test of significance for correlation: Significance of phenotypic, genotypic and environmental correlation coefficients were tested against table value for $\mathrm{r}$ at $(\mathrm{n}-2)$ degrees of freedom from Fischer and Yates (1963) tables where ' $n$ ' denotes total number of entries under study.

\section{Results and Discussion}

The knowledge of inter-character correlation of quantitative characters is useful to design an effective breeding programme. These correlations provide a reliable measure to differentiate the vital association useful in breeding from that of non-vital ones (Falconer, 1981).

The present investigation primarily aimed at assessment of inter-character association between oil and other seed properties viz., fibre properties. Character association was done in all the three experiments comprising of genetic stocks involving both tetraploid $\mathrm{Bt}$ and non-Bt commercial hybrids. The complete set of correlations among seed and oil related parameters are available in the genetic stock.

A perusal of Table 1, 2, 3, 4, 5, 6, and 7 (pooled correlation) indicates absolutely nosignificant association of oil percentage with monopodial branches.

The oil per cent was significantly and positively associated with seed cotton yield at Nagpur and Dharwad but only positive association was observed at Bagalkot. Dani (1984a), Ramalingam (1994) made similar observations.

Plant height showed positive and significant association with oil per cent at Nagpur and Bagalkot. These results completely agreed with Zuquinhao et al., (1995).

Number of sympodial branches was positively correlated with oil per cent at 1 per cent level. Bolls per plant positively and significantly correlated with oil per cent at Nagpur. Twenty boll weight had positive and significant association with oil per cent at Nagpur and Bagalkot. Similar results were reported by Ramalingam et al., (1994). Seed index showed positive and significant correlation with oil per cent. It seems selection for seed index in turn selects for increased oil content. Similar results were observed by Ramalingam et al., (1994). Seed cotton yield positively and significantly correlated with plant height, sympodial branches, bolls per plant, ginning outturn, seed index and oil per cent. Similar observations were reported by Muthu et al., (2004), Nilima et al., (2005), Annapurve et al., (2007) and Ganeshan and Ravindran (2007). Plant height had significant positive association with number of sympodial branches and bolls per plant. 
Table.1 Phenotypic correlations of Bt and non-Bt hybrids for oil and seed cotton yield related traits at Nagpur

\begin{tabular}{|c|c|c|c|c|c|c|c|c|c|c|c|}
\hline Characters & $\begin{array}{c}\text { Seed } \\
\text { cotton } \\
\text { yield }\end{array}$ & $\begin{array}{l}\text { Plant } \\
\text { height }\end{array}$ & $\begin{array}{c}\text { Monopodial } \\
\text { branches }\end{array}$ & $\begin{array}{l}\text { Sympodial } \\
\text { branches }\end{array}$ & $\begin{array}{l}\text { Bolls per } \\
\text { plant }\end{array}$ & $\begin{array}{l}\text { 20-boll } \\
\text { weight }\end{array}$ & $\begin{array}{c}\text { Ginning } \\
\text { outturn } \\
(\%)\end{array}$ & $\begin{array}{l}\text { Seed } \\
\text { index }\end{array}$ & $\begin{array}{c}\text { Oil } \\
\text { content } \\
(\%)\end{array}$ & $\begin{array}{l}\text { Fuzz } \\
(\%)\end{array}$ & $\begin{array}{c}\text { Kernel } \\
(\%)\end{array}$ \\
\hline Seed cotton yield & 1.000 & $0.441 * *$ & -0.009 & $0.350 * *$ & 0.267 & 0.250 & 0.089 & $0.349 *$ & $0.373 * *$ & -0.170 & -0.047 \\
\hline Plant height & & 1.000 & 0.046 & $0.341^{*}$ & $0.408 * *$ & 0.222 & 0.104 & 0.271 & 0.197 & -0.138 & -0.080 \\
\hline Monopodial branches & & & 1.000 & 0.119 & $0.345^{*}$ & 0.183 & 0.166 & $0.308 *$ & 0.116 & 0.041 & 0.002 \\
\hline Sympodial branches & & & & 1.000 & 0.188 & 0.104 & -0.119 & 0.109 & 0.240 & -0.081 & -0.080 \\
\hline Bolls per plant & & & & & 1.000 & -0.035 & 0.128 & $0.331 *$ & $0.312 *$ & 0.101 & -0.149 \\
\hline 20-boll weight & & & & & & 1.000 & -0.077 & $0.381 * *$ & $0.340 *$ & -0.031 & 0.081 \\
\hline Ginning outturn (\%) & & & & & & & 1.000 & -0.058 & -0.069 & -0.005 & -0.065 \\
\hline Seed index & & & & & & & & 1.000 & $0.629 * *$ & -0.064 & 0.090 \\
\hline Oil content (\%) & & & & & & & & & 1.000 & -0.185 & 0.058 \\
\hline Fuzz (\%) & & & & & & & & & & 1.000 & 0.062 \\
\hline Kernel (\%) & & & & & & & & & & & 1.000 \\
\hline
\end{tabular}

*, **: Significant at $5 \%$ and $1 \%$ level of probability

Table.2 Genotypic correlations of Bt and non-Bt hybrids for oil and seed cotton yield related traits at Nagpur

\begin{tabular}{|c|c|c|c|c|c|c|c|c|c|c|c|}
\hline Characters & $\begin{array}{c}\text { Seed } \\
\text { cotton } \\
\text { yield }\end{array}$ & $\begin{array}{c}\text { Plant } \\
\text { height }\end{array}$ & $\begin{array}{l}\text { Monopodial } \\
\text { branches }\end{array}$ & $\begin{array}{c}\text { Sympodial } \\
\text { branches }\end{array}$ & $\begin{array}{l}\text { Bolls per } \\
\text { plant }\end{array}$ & $\begin{array}{l}\text { 20-boll } \\
\text { weight }\end{array}$ & $\begin{array}{c}\text { Ginning } \\
\text { outturn } \\
(\%)\end{array}$ & $\begin{array}{l}\text { Seed } \\
\text { index }\end{array}$ & $\begin{array}{c}\text { Oil } \\
\text { content } \\
(\%)\end{array}$ & $\begin{array}{l}\text { Fuzz } \\
(\%)\end{array}$ & $\begin{array}{c}\text { Kernel } \\
(\%)\end{array}$ \\
\hline Seed cotton yield & 1.000 & $0.571 * *$ & 0.002 & $0.494 * *$ & $0.341 * *$ & $0.355 * *$ & 0.102 & $0.403 * *$ & $0.445 * *$ & -0.183 & -0.086 \\
\hline Plant height & & 1.000 & 0.113 & $0.550 * *$ & $0.512 * *$ & $0.305^{*}$ & 0.241 & $0.455 * *$ & $0.455 * *$ & -0.171 & -0.256 \\
\hline Monopodial branches & & & 1.00 & 0.107 & $0.503 * *$ & 0.096 & 0.237 & $0.365^{*}$ & 0.151 & 0.040 & 0.088 \\
\hline Sympodial branches & & & & 1.000 & $0.623 * *$ & 0.217 & -0.024 & 0.176 & $0.408 * *$ & -0.080 & -0.261 \\
\hline Bolls per plant & & & & & 1.000 & -0.002 & 0.124 & $0.441 * *$ & $0.437 * *$ & 0.131 & -0.232 \\
\hline 20-boll weight & & & & & & 1.000 & -0.037 & $0.497 * *$ & $0.632 * *$ & -0.043 & 0.193 \\
\hline Ginning outturn (\%) & & & & & & & 1.000 & -0.054 & -0.120 & -0.031 & -0.235 \\
\hline Seed index & & & & & & & & 1.000 & $0.795 * *$ & -0.068 & 0.168 \\
\hline Oil content (\%) & & & & & & & & & 1.000 & -0.213 & 0.052 \\
\hline Fuzz (\%) & & & & & & & & & & 1.000 & 0.105 \\
\hline Kernel (\%) & & & & & & & & & & & 1.000 \\
\hline
\end{tabular}

$*, * *$ : Significant at $5 \%$ and $1 \%$ level of probability 
Table.3 Phenotypic correlations of Bt and non-Bt hybrids for oil and seed cotton yield related traits at Dharwad

\begin{tabular}{|c|c|c|c|c|c|c|c|c|c|c|c|}
\hline Characters & $\begin{array}{c}\text { Seed } \\
\text { cotton } \\
\text { yield }\end{array}$ & $\begin{array}{l}\text { Plant } \\
\text { height }\end{array}$ & $\begin{array}{l}\text { Monopodial } \\
\text { branches }\end{array}$ & $\begin{array}{l}\text { Sympodial } \\
\text { branches }\end{array}$ & $\begin{array}{l}\text { Bolls per } \\
\text { plant }\end{array}$ & $\begin{array}{l}\text { 20-boll } \\
\text { weight }\end{array}$ & $\begin{array}{c}\text { Ginning } \\
\text { outturn } \\
(\%)\end{array}$ & $\begin{array}{l}\text { Seed } \\
\text { index }\end{array}$ & $\begin{array}{c}\text { Oil } \\
\text { content } \\
(\%)\end{array}$ & $\begin{array}{c}\text { Fuzz } \\
(\%)\end{array}$ & $\begin{array}{c}\text { Kernel } \\
(\%)\end{array}$ \\
\hline Seed cotton yield & 1.000 & 0.117 & 0.159 & -0.097 & 0.274 & 0.094 & -0.015 & -0.055 & 0.160 & 0.261 & 0.034 \\
\hline Plant height & & 1.000 & 0.078 & 0.274 & 0.156 & 0.085 & 0.296 & -0.231 & -0.051 & -0.007 & -0.026 \\
\hline $\begin{array}{l}\text { Monopodial } \\
\text { branches }\end{array}$ & & & 1.000 & $-0.327^{*}$ & $0.341 *$ & -0.089 & 0.101 & $0.383 * *$ & 0.021 & 0.098 & -0.051 \\
\hline Sympodial branches & & & & 1.000 & 0.050 & -0.032 & 0.121 & $-0.338^{*}$ & -0.007 & -0.045 & 0.051 \\
\hline Bolls per plant & & & & & 1.000 & -0.044 & 0.139 & 0.069 & -0.089 & 0.219 & -0.251 \\
\hline 20-boll weight & & & & & & 1.000 & $-0.333^{*}$ & 0.172 & 0.128 & 0.030 & -0.017 \\
\hline Ginning outturn (\%) & & & & & & & 1.000 & $-0.458 * *$ & -0.186 & -0.003 & -0.017 \\
\hline Seed index & & & & & & & & 1.00 & 0.252 & 0.014 & -0.076 \\
\hline Oil content (\%) & & & & & & & & & 1.000 & 0.064 & 0.073 \\
\hline Fuzz (\%) & & & & & & & & & & 1.000 & 0.103 \\
\hline Kernel (\%) & & & & & & & & & & & 1.000 \\
\hline
\end{tabular}

$*, * *$ : Significant at $5 \%$ and $1 \%$ level of probability

Table.4 Genotypic correlations of Bt and non-Bt hybrids for oil and seed cotton yield related traits at Dharwad

\begin{tabular}{|c|c|c|c|c|c|c|c|c|c|c|c|}
\hline Characters & $\begin{array}{c}\text { Seed } \\
\text { cotton } \\
\text { yield }\end{array}$ & $\begin{array}{c}\text { Plant } \\
\text { height }\end{array}$ & $\begin{array}{c}\text { Monopodial } \\
\text { branches }\end{array}$ & $\begin{array}{l}\text { Sympodial } \\
\text { branches }\end{array}$ & $\begin{array}{l}\text { Bolls per } \\
\text { plant }\end{array}$ & $\begin{array}{l}\text { 20-boll } \\
\text { weight }\end{array}$ & $\begin{array}{c}\text { Ginning } \\
\text { outturn } \\
(\%)\end{array}$ & $\begin{array}{l}\text { Seed } \\
\text { index }\end{array}$ & $\begin{array}{c}\text { Oil } \\
\text { content } \\
(\%)\end{array}$ & $\begin{array}{c}\text { Fuzz } \\
(\%)\end{array}$ & $\begin{array}{c}\text { Kernel } \\
(\%)\end{array}$ \\
\hline Seed cotton yield & 1.000 & 0.179 & 0.139 & -0.125 & $0.448 * *$ & -0.038 & $0.356 * *$ & -0.158 & $0.356 * *$ & $0.632 * *$ & 0.111 \\
\hline Plant height & & 1.000 & 0.121 & $0.453 * *$ & $0.340 *$ & -0.055 & $0.460 * *$ & $-0.349 *$ & -0.072 & 0.035 & -0.111 \\
\hline Monopodial branches & & & 1.000 & -0.139 & $0.964 * *$ & -0.179 & 0.223 & $0.537 * *$ & 0.253 & 0.291 & -0.223 \\
\hline Sympodial branches & & & & 1.000 & -0.181 & -0.053 & $0.299 *$ & $-0.632 * *$ & $-0.364 * *$ & $-0.320 *$ & 0.259 \\
\hline Bolls per plant & & & & & 1.000 & -0.131 & $0.309 *$ & 0.216 & -0.021 & $0.414 * *$ & -0.198 \\
\hline 20-boll weight & & & & & & 1.000 & $-0.317^{*}$ & $0.305^{*}$ & 0.095 & 0.206 & $-0.303 *$ \\
\hline Ginning outturn (\%) & & & & & & & 1.000 & $-0.593 * *$ & $-0.497 * *$ & -0.069 & -0.155 \\
\hline Seed index & & & & & & & & 1.000 & $0.408 * *$ & 0.062 & -0.084 \\
\hline Oil content (\%) & & & & & & & & & 1.000 & 0.142 & 0.204 \\
\hline Fuzz $(\%)$ & & & & & & & & & & 1.000 & 0.094 \\
\hline Kernel (\%) & & & & & & & & & & & 1.000 \\
\hline
\end{tabular}

*, **: Significant at $5 \%$ and $1 \%$ level of probability 
Table.5 Phenotypic correlations of Bt and non-Bt hybrids for oil and seed cotton yield related traits at Bagalkot

\begin{tabular}{|c|c|c|c|c|c|c|c|c|c|c|c|}
\hline Characters & $\begin{array}{c}\text { Seed } \\
\text { cotton } \\
\text { yield }\end{array}$ & $\begin{array}{l}\text { Plant } \\
\text { height }\end{array}$ & $\begin{array}{c}\text { Monopodial } \\
\text { branches }\end{array}$ & $\begin{array}{l}\text { Sympodial } \\
\text { branches }\end{array}$ & $\begin{array}{l}\text { Bolls per } \\
\text { plant }\end{array}$ & $\begin{array}{l}\text { 20-boll } \\
\text { weight }\end{array}$ & $\begin{array}{c}\text { Ginning } \\
\text { outturn } \\
(\%)\end{array}$ & $\begin{array}{l}\text { Seed } \\
\text { index }\end{array}$ & $\begin{array}{c}\text { Oil } \\
\text { content } \\
(\%)\end{array}$ & $\begin{array}{l}\text { Fuzz } \\
(\%)\end{array}$ & $\begin{array}{c}\text { Kernel } \\
(\%)\end{array}$ \\
\hline Seed cotton yield & 1.000 & 0.091 & 0.231 & -0.056 & -0.123 & 0.0159 & -0.109 & 0.272 & 0.234 & -0.149 & 0.095 \\
\hline Plant height & & 1.000 & $0.348 *$ & 0.040 & $0.321 * *$ & 0.114 & $-0.297 *$ & 0.079 & $0.356 * *$ & 0.003 & 0.107 \\
\hline Monopodial branches & & & 1.000 & 0.255 & $0.292 *$ & 0.010 & $-0.386 * *$ & 0.172 & $0.353 * *$ & -0.113 & 0.039 \\
\hline Sympodial branches & & & & 1.000 & 0.249 & 0.021 & -0.034 & 0.165 & 0.161 & -0.030 & 0.112 \\
\hline Bolls per plant & & & & & 1.000 & -0.268 & -0.097 & 0.025 & 0.069 & 0.116 & 0.225 \\
\hline 20-boll weight & & & & & & 1.000 & -0.003 & 0.132 & $0.282 *$ & -0.019 & -0.025 \\
\hline Ginning outturn (\%) & & & & & & & 1.000 & -0.025 & $-0.360 * *$ & 0.080 & -0.141 \\
\hline Seed index & & & & & & & & 1.000 & 0.268 & -0.057 & 0.112 \\
\hline Oil content (\%) & & & & & & & & & 1.000 & -0.116 & -0.033 \\
\hline Fuzz (\%) & & & & & & & & & & 1.000 & -0.031 \\
\hline Kernel (\%) & & & & & & & & & & & 1.000 \\
\hline
\end{tabular}

*, **: Significant at $5 \%$ and $1 \%$ level of probability

Table.6 Genotypic correlations of Bt and non-Bt hybrids for oil and seed cotton yield related traits at Bagalkot

\begin{tabular}{|c|c|c|c|c|c|c|c|c|c|c|c|}
\hline Characters & $\begin{array}{c}\text { Seed } \\
\text { cotton } \\
\text { yield }\end{array}$ & $\begin{array}{l}\text { Plant } \\
\text { height }\end{array}$ & $\begin{array}{c}\text { Monopodial } \\
\text { branches }\end{array}$ & $\begin{array}{l}\text { Sympodial } \\
\text { branches }\end{array}$ & $\begin{array}{l}\text { Bolls per } \\
\text { plant }\end{array}$ & $\begin{array}{l}\text { 20-boll } \\
\text { weight }\end{array}$ & $\begin{array}{c}\text { Ginning } \\
\text { outturn } \\
(\%)\end{array}$ & $\begin{array}{l}\text { Seed } \\
\text { index }\end{array}$ & $\begin{array}{c}\text { Oil } \\
\text { content } \\
(\%)\end{array}$ & $\begin{array}{c}\text { Furzz } \\
(\%)\end{array}$ & $\begin{array}{c}\text { Kernel } \\
(\%)\end{array}$ \\
\hline Seed cotton yield & 1.000 & 0.052 & 0.270 & -0.270 & -0.111 & 0.225 & -0.184 & $0.355 * *$ & 0.259 & -0.214 & 0.016 \\
\hline Plant height & & 1.000 & $0.423 * *$ & 0.012 & $0.371 * *$ & 0.205 & $-0.373 * *$ & 0.105 & $0.389 * *$ & 0.042 & 0.211 \\
\hline Monopodial branches & & & 1.000 & $0.495 * *$ & $0.335^{*}$ & 0.086 & $-0.505 * *$ & 0.209 & $0.423 * *$ & -0.156 & 0.036 \\
\hline Sympodial branches & & & & 1.000 & $0.300^{*}$ & -0.229 & -0.103 & $0.297 *$ & 0.163 & $0.273^{*}$ & 0.311* \\
\hline Bolls per plant & & & & & 1.000 & $-0.292^{*}$ & -0.141 & -0.030 & 0.076 & 0.240 & $0.602 * *$ \\
\hline 20-boll weight & & & & & & 1.000 & -0.119 & 0.259 & $0.537 * *$ & 0.261 & $-0.371 * *$ \\
\hline Ginning outturn (\%) & & & & & & & 1.000 & -0.094 & $-0.507 * *$ & 0.015 & -0.250 \\
\hline Seed index & & & & & & & & 1.000 & $0.337 * *$ & -0.156 & 0.271 \\
\hline Oil content $(\%)$ & & & & & & & & & 1.000 & -0.166 & -0.042 \\
\hline Fuzz $(\%)$ & & & & & & & & & & 1.00 & -0.210 \\
\hline Kernel (\%) & & & & & & & & & & & 1.000 \\
\hline
\end{tabular}

$*, * *$ : Significant at $5 \%$ and $1 \%$ level of probability 
Table.7 Pooled coefficient of correlation across 48 commercial Bt and non-Bt genetic stock for seed oil and yield related traits

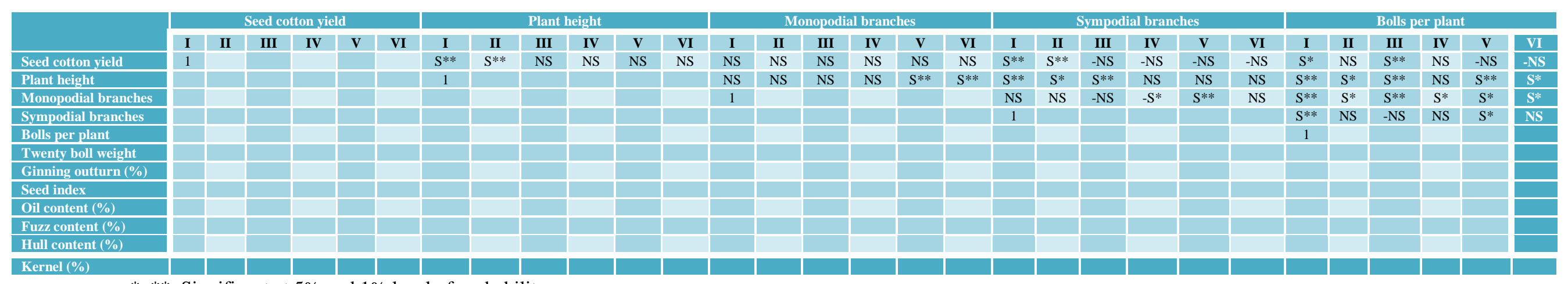
*,*: Significant at $5 \%$ and $1 \%$ leve
II - Phenotypic correlation (Nagpur)
III - Genotypic correlation (Dharwad)
IV - Phenotypic correlation (Dharwad)
V - Genotypic correlation (Bagalkot)
VI - Phenotypic correlation (Bagalkot)

Contd........

\begin{tabular}{|c|c|c|c|c|c|c|c|c|c|c|c|c|c|c|c|c|c|c|c|c|c|c|c|c|}
\hline & \multicolumn{6}{|c|}{ Twenty boll weight } & \multicolumn{6}{|c|}{ Ginning outturn (\%) } & \multicolumn{6}{|c|}{ Seed index (\%) } & \multicolumn{6}{|c|}{ Oil content (\%) } \\
\hline & I & II & III & IV & $\mathbf{v}$ & VI & I & II & III & IV & v & VI & I & II & III & IV & $\mathbf{v}$ & VI & I & II & III & IV & v & VI \\
\hline Seed cotton yield & $\mathrm{S}^{*}$ & NS & - NS & NS & NS & NS & NS & NS & $\mathrm{S}^{* *}$ & $-\mathrm{NS}$ & -NS & - NS & $\mathrm{S}^{* *}$ & $\mathrm{~S}^{*}$ & - NS & $-\mathrm{NS}$ & $\mathrm{S}$ & NS & $\mathrm{S}^{* *}$ & $\mathrm{~S}^{* *}$ & $\mathrm{~S}^{* *}$ & NS & NS & NS \\
\hline Plant height & $\mathrm{S}^{*}$ & NS & - NS & NS & NS & NS & NS & NS & $S^{* *}$ & NS & $-S^{* *}$ & - NS & $S^{* *}$ & NS & $-5^{*}$ & $-\mathrm{NS}$ & NS & NS & $\mathrm{S}^{* *}$ & NS & $-\mathrm{NS}$ & $-\mathrm{NS}$ & NS & $S^{* * *}$ \\
\hline Monopodial branches & NS & NS & - NS & - NS & NS & NS & NS & NS & NS & NS & $-S^{* *}$ & $-S^{* *}$ & $\mathrm{~S}^{*}$ & $\mathrm{~S}^{*}$ & $5^{* *}$ & $5^{* *}$ & NS & NS & NS & NS & NS & NS & NS & $\mathrm{S}^{*}$ \\
\hline Sympodial branches & NS & NS & $-\mathrm{NS}$ & - NS & $-\mathrm{NS}$ & NS & $-\mathrm{NS}$ & $-\mathrm{NS}$ & $\mathrm{S}^{*}$ & NS & $-\mathrm{NS}$ & $-\mathrm{NS}$ & NS & NS & $-5 * *$ & $-5^{*}$ & $\mathrm{~S}^{*}$ & NS & $\mathrm{S}^{* *}$ & NS & $-\mathrm{S}^{* *}$ & $-\mathrm{NS}$ & NS & NS \\
\hline Bolls per plant & -NS & $-\mathrm{NS}$ & $-\mathrm{NS}$ & - NS & - NS & - NS & NS & NS & $\mathrm{S}^{*}$ & NS & $-\mathrm{NS}$ & $-\mathrm{NS}$ & $\mathrm{S}^{* * *}$ & $\mathrm{~S}^{*}$ & NS & NS & - NS & NS & $\mathrm{S}^{* *}$ & NS & $-\mathrm{NS}$ & - NS & NS & NS \\
\hline Twenty boll weight & & 1 & & & & & - NS & - NS & $-\mathrm{S}^{*}$ & $-5^{*}$ & $-\mathrm{NS}$ & $-\mathrm{NS}$ & $\mathrm{S}^{* *}$ & $\mathrm{~S}^{* *}$ & $5^{*}$ & NS & NS & NS & $\mathrm{S}^{* *}$ & NS & NS & NS & $\mathrm{S}^{* *}$ & $\mathrm{~S}^{*}$ \\
\hline Ginning outturn (\%) & & & & & & & 1 & & & & & & - NS & - NS & $-5 * *$ & $-5 * *$ & - NS & - NS & - NS & - NS & $-S^{* * *}$ & - NS & $-S^{* *}$ & $-\mathrm{S} * * *$ \\
\hline Seed index & & & & & & & & & & & & & 1 & & & & & & $\mathrm{~S}^{* * *}$ & $\mathrm{~S}^{* *}$ & $\mathrm{~S}^{* *}$ & NS & $\mathrm{S}^{* *}$ & NS \\
\hline Oil content $(\%)$ & & & & & & & & & & & & & & & & & & & 1 & & & & & \\
\hline Fuzz content (\%) & & & & & & & & & & & & & & & & & & & & & & & & \\
\hline Hull content (\%) & & & & & & & & & & & & & & & & & & & & & & & & \\
\hline Kernel (\%) & & & & & & & & & & & & & & & & & & & & & & & & \\
\hline
\end{tabular}

*, **: Significant at $5 \%$ and $1 \%$ level of probability 
Contd.......

\begin{tabular}{|c|c|c|c|c|c|c|c|c|c|c|c|c|c|c|c|c|c|c|}
\hline & \multicolumn{6}{|c|}{ Fuzz content (\%) } & \multicolumn{6}{|c|}{ Hull content (\%) } & \multicolumn{6}{|c|}{ Kernel percentage } \\
\hline & I & II & III & IV & $\mathbf{V}$ & VI & $\mathbf{I}$ & II & III & IV & $\mathbf{V}$ & VI & I & II & III & IV & $\mathbf{V}$ & VI \\
\hline Seed cotton yield & NS & $-\mathrm{NS}$ & $\mathrm{S}^{*}$ & NS & $-\mathrm{NS}$ & $-\mathrm{NS}$ & NS & NS & - NS & - NS & - NS & - NS & - NS & - NS & NS & NS & NS & NS \\
\hline Plant height & - NS & - NS & NS & - NS & NS & NS & NS & NS & NS & NS & - NS & - NS & - NS & - NS & - NS & - NS & NS & $\mathbf{N S}$ \\
\hline Monopodial branches & NS & NS & NS & NS & - NS & $-\mathrm{NS}$ & NS & NS & NS & NS & - NS & - NS & NS & NS & - NS & $-\mathrm{NS}$ & NS & NS \\
\hline Sympodial branches & - NS & - NS & $-S^{*}$ & $-\mathrm{NS}$ & $S^{*}$ & $-\mathrm{NS}$ & - NS & NS & - NS & - NS & $S^{*}$ & - NS & - NS & - NS & NS & NS & $S^{*}$ & $\overline{N S}$ \\
\hline Bolls per plant & NS & NS & $\mathrm{S}^{* *}$ & NS & NS & NS & - NS & NS & NS & NS & $-S^{* *}$ & - NS & - NS & - NS & - NS & - NS & $\mathrm{S}^{* *}$ & NS \\
\hline Twenty boll weight & - NS & - NS & - NS & - NS & NS & NS & NS & - NS & $\mathrm{S}^{*}$ & NS & $\mathrm{S}^{* * *}$ & NS & NS & NS & $-\mathrm{S}^{*}$ & - NS & $\mathrm{S} * *$ & $-\mathbf{N S}$ \\
\hline Ginning outturn (\%) & - NS & - NS & - NS & - NS & NS & NS & - NS & NS & NS & NS & NS & NS & - NS & - NS & - NS & - NS & - NS & $-\mathbf{N S}$ \\
\hline Seed index & $-\mathrm{NS}$ & $-\mathrm{NS}$ & NS & NS & - NS & $-\mathrm{NS}$ & NS & - NS & NS & NS & $-\mathrm{NS}$ & $-\mathrm{NS}$ & NS & NS & $-\mathrm{NS}$ & $-\mathrm{NS}$ & NS & NS \\
\hline Oil content (\%) & - NS & - NS & NS & NS & - NS & $-\mathrm{NS}$ & - NS & - NS & $-\mathrm{NS}$ & - NS & NS & NS & NS & NS & NS & NS & - NS & NS \\
\hline Fuzz content (\%) & 1 & & & & & & - NS & - NS & - NS & - NS & NS & NS & NS & NS & NS & NS & - NS & $-\mathrm{NS}$ \\
\hline Hull content (\%) & & & & & & & 1 & & & & & & $-S^{* *}$ & $-\mathrm{S}^{* *}$ & $-\mathrm{S}^{* *}$ & $-\mathrm{S}^{* *}$ & $-\mathrm{S}^{* *}$ & $-S * *$ \\
\hline Kernel (\%) & & & & & & & & & & & & & 1 & & & & & \\
\hline
\end{tabular}




\section{Correlation coefficient}

\begin{tabular}{|l|c|c|c|}
\hline Source of variation & d.f. & MSP & Expectation \\
\hline Replication & $(\mathrm{r}-1)$ & RMSP & \\
\hline Treatment & $(\mathrm{t}-1)$ & TMSP & $\mathrm{\sigma e}^{2}+\mathrm{ro}^{2} \mathrm{~g}$ \\
\hline Error & $(\mathrm{r}-1)(\mathrm{t}-1)$ & EMSP & $\sigma^{2} \mathrm{e}^{2}$ \\
\hline \multicolumn{1}{|c|}{ Total } & $(\mathrm{rt}-1)$ & & \\
\hline
\end{tabular}

Similar results were reported by Ganeshan and Ravindran (2007). Monopodial branches were significantly and positively correlated with bolls per plant in all the locations. Boll weight showed significant positive association with seed index at Nagpur and Dharwad. Similar results were got by Nilima et al., (2005). With a special reference to oil content, the present study revealed that selecting for higher seed cotton yield, plant height, more number of sympodial branches, higher seed index and higher boll weight leads to increased oil content in the seed.

Overall considering the correlation of seed cotton yield and oil content with other important traits the following conclusion can be drawn. Seed cotton yield showed positive and significant association with oil content at two locations viz., Nagpur and Dharwad. Selecting for higher seed cotton yield would also help in increased oil content. The oil content expressed positive and significant association with seed index with all three locations viz., Nagpur, Dharwad and Bagalkot. Therefore, seed index can be used to select for high oil content. Oil content also exhibited positive and significant correlation with twenty boll weight and plant height at two location viz., Nagpur and Bagalkot. However, oil content expressed negative and significant association with ginning outturn at two location viz., Dharwad and Bagalkot.

The oil per cent positively correlated with seed cotton yield, plant height, sympodial branches, seed cotton yield bolls per plant, ginning out turn and seed index. The study clearly showed that if increased yield and yield related traits with help of breeding strategy in turns it will increase oil content or vice versa.

\section{References}

Annapurve, S. N., Deshmukh, G. S., Kausale, S. P. and Kale, U. V., 2007, Correlation study of yield contributing characters in American cotton. J. Cotton Res. Dev., 21(1): 16-20.

Dani, R. G., 1984a, Variability of seed oil and productivity in some Indian cottons tested in Texas. Indian J. Agric. Sci., 54: 550-556.

Falconer, D. S., 1981, Introduction to Quantitative Genetics - English Language Book Society, Longman, pp. 100-169.

Falconer, D. S., 1981, Introduction to Quantitative Genetics - English Language Book Society, Longman, pp. 100-169.

Ganeshan, K. N. and Raveendran, T. S., 2007, Significance of yield contributing traits in enhancing the yield of American cotton (Gossypium hirsutum L.). J. Indian Soc. Cotton Improv., 32: 50-57.

Hayes, H. K., Immer, I. R. and Smith, D. C., 1955, Methods of Plant Breeding, McGraw Hill Company, Inc.

Jambunathan, R., Raju, S. M. and Barde, S. P., 1985, Analysis of oil content of groundnuts by nuclear magnetic resonance spectrophotometry. J. Food Sci., Agril., 36: 162-166. 
Muthu, R., Kandasamy, G. and Jayaramachandran, 2004, Correlation and path coefficient analysis for yield and fibre quality traits in cotton ( $G$. hirsutum L.). Indian Soc. Cotton. Improv., 74: 17-20.

Neelima, S., Chengareddy, and Narsi Reddy, 2005, Association and path analysis in American cotton (Gossypium hirsutum L.). J. Indian Soc. Cotton Improv., 30: 53-58.

Ramalingam, A., Sivasamy, N., Koodlingam, K. and Subramanian, S., 1994,
Association of seed oil components and path coefficient analysis in cotton. $J$. Indian Soc. Cotton Improv., 19(2): 146150.

Singh, R. K. and Chaudhary, B. D., 1977, Biometric Methods in Quantitative Genetics Analysis, Kalyani Publishers, New Delhi.

Zhuquianhao, Xufahua and Yubixya, 1995, Genetic analysis of heterosis for cotton seed quality traits in glandless cotton. Acta Agriculture Zhe Jaingensis. 621628.

\section{How to cite this article:}

Nagappa Harijan and Khadi, B.M. 2018. Correlation Co-Efficient Studies of Oil, Yield and Related Traits in Bt and Non Bt Hybrids. Int.J.Curr.Microbiol.App.Sci. 7(03): 84-93. doi: https://doi.org/10.20546/ijcmas.2018.703.010 